Convergences francophones 6.3 (2021): 29-40

http://mrujs.mtroyal.ca/index.php/cf/index

\title{
Les Inversions de l'héritage littéraire entre Eric-Emmanuel Schmitt et Romain Gary
}

\author{
Molleen Shilliday \\ University of the Fraser Valley, Canada
}

Chaque référence faite à La Vie devant soi (1975) de Romain Gary dans Monsieur Ibrahim et les fleurs du Coran (2001) d'Eric-Emmanuel Schmitt retentit chez le lecteur comme une belle phrase de Proust chez n'importe quel écrivain contemporain. Si pour certains il s'agit d'une relation inégale, elle reste toutefois enrichissante et même nécessaire car elle repose sur le rapport entre les œuvres contemporaines et celles relevant du patrimoine littéraire et les enjeux sont patrimoniaux et esthétiques : Schmitt rend hommage à Gary tout en mettant en lumière les déficiences potentielles de son propre texte. Pourquoi s'ouvrir à ce genre de comparaison? Gary, après tout, est le seul écrivain a gagné le prix Goncourt deux fois, et La Vie devant soi, publié sous le nom de plume Emile Ajar, reste le symbole de l'ingéniosité de cet écrivain qui a trompé tout un monde critique. Ce fait représente peut-être la clé de ce choix de père/paire littéraire. La rebuffade académique et critique de cette ruse a été immédiate et Gary est resté relégué à la périphérie des grands écrivains pendant plusieurs décennies. Ce n'est qu'au vingt-et-unième siècle que l'intérêt pour son œuvre augmente de manière significative -des colloques ainsi que des ouvrages lui sont dédiés. Son absence de la bibliothèque de la Pléiade, jusqu'en 2019, contrairement à ses contemporains (tels que Camus, Duras, Sartre et Simon), demeure toutefois révélatrice des séquelles de son subterfuge identitaire.

Or, en 2001, Schmitt nous rappelle l'influence littéraire d'un écrivain souvent occulté par son mépris des règles, les péripéties de sa vie personnelle et son suicide. Cet hommage se défie de la classification : la singularité du style de Schmitt nie le pastiche et les inversions diégétiques astucieuses contournent la réécriture. Alors que le lignage biologique entre enfant et parent est redéfini dans les deux romans, le lignage littéraire établi entre les deux écrivains semble subrepticement promouvoir un certain genre romanesque remettant en question l'ère post et substitue à la rupture héréditaire une filiation paradoxalement autre et même. Selon Dominique Viart, «... il ne s'agit plus aujourd'hui d'inventer du neuf mais de travailler avec ce dont la culture et la pensée héritent ... la fin du $20^{\mathrm{e}}$ siècle est consciente du point ultime atteint par la modernité » (25). La simplicité du style de Schmitt donne à son court roman le caractère d'un texte seuil : il demeure entièrement accessible au lecteur qui cherche un roman émouvant, tout en dissimulant un système complexe de signes et de symboles emprunté à son prédécesseur qu'un lecteur perspicace peut apprécier. En effet, dans Monsieur Ibrahim et les fleurs du Coran, Schmitt valorise les inversions et les oppositions qui servent à déconstruire les frontières entre soi et l'autre et à créer une filiation de la différence entre son texte et La Vie devant soi, entre lui-même et Romain Gary. Ce sont surtout l'éloge de l'humanité de ceux qui demeurent à la marge et le plaidoyer de l'amour qui lient ces deux livres et motivent notre intérêt critique. 
Convergences francophones 6.3 (2021): 29-40

http://mrujs.mtroyal.ca/index.php/cf/index

Les trames narratives dans Monsieur Ibrahim et les fleurs du Coran renvoient inlassablement au texte d'origine, mettant le lecteur sur la bonne piste pour découvrir la beauté de la filiation littéraire. Dès l'incipit, Schmitt établit le lien entre son petit livre de 85 pages publié chez Albin Michel en 2001 et celui de Gary, 274 pages, publié chez Mercure de France en 1975. Dans les deux romans, l'enfantnarrateur, Momo, est rejeté par sa famille et forge une amitié avec un homme âgé qui comble le vide parental. Le choix du prénom, Momo, constitue l'inversion centrale de l'histoire. Chez Gary, il s'agit de Mohammed, surnommé Momo, un beau garçon arabe et musulman qui habite à la pension gérée par Madame Rosa. Chez Schmitt, Momo est le sobriquet de Moïse, un jeune garçon juif qui habite avec son père distant et sévère. Alors que le surnom du jeune garçon reste le même chez Schmitt, le changement du prénom transforme l'allusion religieuse. Par conséquent, les grands prophètes du judaïsme et de l'islam sont rapprochés à travers ces deux jeunes garçons immatures et capricieux. Le sens de l'humour des deux écrivains est à la fois narquois et jovial, diminuant la toute-puissance des symboles religieux et valorisant l'histoire d'un enfant rebelle mal aimé. Par ailleurs, dans les deux romans, l'adulte qui adopte Momo pratique une religion qui s'oppose historiquement à celle de l'enfant. Cette relation centrale dévoile effectivement la véritable proximité des deux religions et les qualités humaines, telles que l'amour et l'amitié, qui peuvent éclipser l'intolérance et la haine religieuses. Chez Gary, Momo/Mohammed devient le fils de Madame Rosa, une vieille juive traumatisée par la Shoah. Chez Schmitt, Moïse/Momo passe son temps avec l'épicier du coin, Monsieur Ibrahim qui est soufi pratiquant du Croissant d'Or. A travers l'opposition religieuse parent/enfant, Gary et Schmitt imprègnent le texte d'humour, allégeant ainsi la charge des thèmes plus lourds : le racisme, la vulnérabilité des êtres marginalisés, le traumatisme des survivants, le vieillissement, la mort et le deuil. Toutefois, la façon dont ces deux écrivains abordent ces sujets difficiles s'oppose. D'un côté, Schmitt semble vouloir protéger son lecteur des émotions trop crues. Les actes violents ont lieu hors champ et sont décrits après l'événement, au cours du dialogue. Ce style facilite le travail du lecteur en faisant appel à sa compassion et à sa sympathie. De l'autre côté, l'humour de Gary se retrouve dans l'image décrite par le témoin. L'image est vive et parfois même grotesque, ce qui ajoute une certaine vulnérabilité à la scène et suscite l'empathie du lecteur.

L'empathie est inextricablement liée à l'expérience et notre capacité à accéder à ce niveau de compréhension humaine est souvent nourrie par une représentation visuelle de la souffrance de l'autre et du corps souffrant. Dans $L a$ Vie devant soi, Gary s'affranchit des règles normatives de la pudeur et de la modestie en ce qui concerne la souffrance corporelle, suscitant effectivement l'empathie du lecteur par le biais du malaise engendré. Par conséquent, le malheur et la douleur semblent transcender la page. Paradoxalement, c'est le côté grotesque qui humanise ses personnages : «... Madame Rosa, avec tous ces kilos qu'elle portait sur elle et seulement deux jambes, c'était une vraie source de vie quotidienne, avec tous les soucis et les peines. » (Gary 9). Le corps de Madame Rosa prend toute la place et Momo, narrateur fidèle, peint un portrait qui ne cache rien : « elle n'avait pour ainsi dire plus de cheveux et pesait dans les quatre-vingt- 
Convergences francophones 6.3 (2021): 29-40

http://mrujs.mtroyal.ca/index.php/cf/index

quinze kilos, tous les uns plus moches que les autres » (116). La franchise innocente de Momo est hilarante, il remarque les détails les plus anodins. Il décrit son « cul plus grand que d'habitude » (36), sa laideur qui se cache plutôt bien quand elle est triste (23), le « racolage avec ses yeux et les trucs avec ses lèvres » qu'elle fait quand elle oublie son âge redevenant momentanément la prostituée coquette qu'elle était jadis (140).

Si l'humour de Gary frôle le grotesque, il s'agit toutefois d'un grotesque réel qui souligne les conséquences physiques et psychologiques de l'exil. L'humour grotesque s'accompagne du retour du refoulé. Ce rare appariement permet à Gary de mettre le jeune narrateur en position de témoin, de montrer les dégâts que produisent la marginalisation et le traumatisme sans avoir constamment recours au ressassement du passé :

Elle était à poil au milieu de la pièce, en train de s'habiller pour aller au boulot, comme lorsqu'elle se défendait encore. Bon moi j'ai rien vu dans ma vie et j'ai pas tellement le droit de dire ce qui est effrayant et ce qui ne l'est pas plus qu'autre chose, mais je vous jure que Madame Rosa à poil, avec des bottes de cuir et des culottes noires en dentelles autour du cou, parce qu'elle s'était trompée de côté, et des niches comme ça dépasse l'imagination ... . Madame Rosa essayait de remuer le cul comme dans un sex-shop, mais comme chez elle le cul dépassait les possibilités humaines...siyyid! Je crois que c'était la première fois que j'ai murmuré une prière, celle pour les mahboûl, mais elle a continué à se tortiller avec un petit sourire coquin et une chatte comme je ne le souhaite à personne. Je comprenais bien que c'était chez elle l'effet du choc récapitulatif .... (161)

Cette scène où le corps devient le véhicule métonymique de la déchéance et de la sénilité de Madame Rosa, représente une autre opposition dans le texte. La mémoire de Madame Rosa s'efface rapidement et s'accroche aux moments déterminants de sa vie, aux moments de grand bonheur et de souffrance extrême. Dans ses dentelles et ses bottes, Madame Rosa revit ses moments de gloire, de beauté et de coquetterie avec plaisir et sans gêne. Ce qui est singulier ici est la façon dont le burlesque et le grotesque fonctionnent comme une sorte d'écran chinois à travers lequel nous pouvons déchiffrer les ombres qui assombrissent la mémoire de Madame Rosa. Cette scène, quoique drôle, évoque les autres épisodes où le retour du refoulé interrompt le présent et Madame Rosa revit sa déportation et son internement à Auschwitz. Gary évoque implicitement le traumatisme primaire sans y faire référence explicitement. Il s'agit d'un rappel tacite de la complexité de la mémoire et de l'incompréhensibilité de la souffrance d'une victime de la Shoah. Il s'agit aussi d'une manière de favoriser l'empathie à travers le grotesque et le rire. Le rire devance le jugement, humanise l'image et on contemple l'Autre en le voyant pleinement.

D'autres repères thématiques scintillent également au fond du passage cité créant une cohésion esthétique et remettant au cœur du récit les valeurs humanistes de Gary. Dans ce passage, la différence entre les personnages se manifeste à travers la subtilité langagière. L'usage des termes arabes « siyyid »et « mahboûl » souligne 
Convergences francophones 6.3 (2021): 29-40

http://mrujs.mtroyal.ca/index.php/cf/index

l'écart religieux et ethnique qui divise normalement juifs et musulmans, Israélites et Magrébines mais qui relie paradoxalement Madame Rosa et Momo. Reconnaissant l'importance de l'héritage et d'un sentiment d'appartenance, Madame Rosa a assuré l'éducation musulmane de Momo. Il prononce par la suite une prière musulmane pour elle. Nous verrons que ce « dialogue interreligieux » (Illman) est également un aspect fondamental de l'écriture de Schmitt. En ce qui concerne le couple mal assorti de Gary, tout ce qui a trait à la différence raciale, ethnique ou religieuse se voit détourné de son courant habituel (l'intolérance et la haine) vers l'humanisme, l'empathie et l'amour. Bien que la voix de l'écrivain s'éclipse souvent grâce à la singularité de la voix narrative, l'expression « choc récapitulatif» dévoile ici sa présence et son espoir de mettre en lumière la gravité des problèmes psychologiques dont souffrent les personnes traumatisées.

Par ailleurs, l'humour de Gary s'inscrit dans une longue lignée littéraire. Par exemple, son humour évoque les machinations du théâtre de l'absurde, la danse de Vénus de Caligula dans Caligula (Camus), la métamorphose de Jean dans Rhinocéros (Ionesco), les simulacres pornographies du Balcon (Genet). Grotesque et théâtrale, cette scène (et le roman en général) met en œuvre un système de double signification où l'humour subvertit les valeurs de la société et souligne les failles du système français. Ce n'est qu'une accumulation de problèmes historiques, sociaux, culturels qui ont conduit Momo devant une telle scène. La France néglige le bien être des personnes vulnérables vivant dans les marges. L'angoisse côtoie Madame Rosa et les orphelins sous sa charge à cause de la menace que pose sa santé défaillante. Le message sous-jacent est dérisoire. Après avoir témoigné de la nudité sénile de Madame Rosa, Momo se précipite dehors, se met à courir, mais «ce n'était pas pour [se] sauver, ça n'existe pas » (Gary 161).

A travers La Vie devant soi, Gary esthétise ainsi l'importance du témoignage et de l'ouverture. Madame Rosa souffre d'une profonde peur associée à ses expériences durant la Deuxième Guerre mondiale. La seule chose qui soulage ses moments d'inquiétude incontrôlable est de se cacher à la cave. Elle a muni cette cachette de provisions, un lit bon à jeter et une menora : elle l'appelle son « trou juif ». La première fois que le petit Momo est le témoin de cette descente à la cave, il ne peut s'empêcher de la suivre. " J'avais les genoux qui tremblaient et c'était terrible de voir cette Juive qui descendait les étages avec des ruses de Sioux comme si c'était plein d'ennemis et encore pire » (37). La peur de Madame Rosa se transmet au petit garçon, mais il ne prend pas la fuite : il descend et se trouve face au traumatisme secret de la vieille survivante. Au moment où il traverse le seuil, il dit : "J'ai regardé » (38). Cette courte phrase représente non seulement une reconnaissance du mal vécu, mais aussi une affirmation du rôle important $\mathrm{du}$ témoin. Momo décrit la salle en détails et déclare subitement : «je n'avais plus peur, sauf que j'avais le cul nu et je commençais à me sentir froid » (38). On constate que l'incompréhension du garçon permet à l'écrivain de mêler le comique et le tragique. La scène se termine avec la phrase suivante : "Quand elle a remonté, elle n'avait plus peur et moi non plus, parce que c'est contagieux » (39). La descente à la cave n'est pas un leitmotiv rare dans les textes portant sur la guerre. Cette descente est souvent liée à la folie, comme dans Hiroshima mon amour de 
Convergences francophones 6.3 (2021): 29-40

http://mrujs.mtroyal.ca/index.php/cf/index

Marguerite Duras, ou aux bombardements, comme dans Persepolis de Marjane Satrapi ou plus récemment All the light we cannot see d'Anthony Doerr. Dans ces textes, la descente communique l'impensable et l'indicible des expériences inassimilables et le lecteur saisit la peur de la victime sans vraiment la partager. La singularité de la descente à la cave dans La Vie devant soi découle de la perspective. Cette scène est narrée et partagée par un personnage d'une autre génération et d'une autre culture, ce qui donne à la descente mémorielle un aspect cathartique et atemporel. Le lecteur comprend que lui aussi est responsable du passé sur lequel le récit s'érige.

L'obésité, la mémoire et la déchéance de Madame Rosa s'opposent à la petite taille, à l'innocence, à la beauté et à la jeunesse du narrateur : il pique une crise lorsqu'il se fait soulever sur les genoux d'un étranger et il rêve d'être un grand policier musclé pour ne plus avoir peur. La beauté de Momo se fait même remarquer par des étrangers $(81,98)$ et il promet à Madame Rosa de ne jamais vendre son corps. Cette inversion entre les deux personnages prépare le lecteur au moment culminant où Momo affrontera la vie sans Madame Rosa. La notion du retour en arrière, déjà souligné par le concept de " choc récapitulatif», devient un mécanisme d'adaptation pour le narrateur. Par exemple, Momo entre par hasard dans une salle de doublage où les scènes d'un film reculent lorsqu'il appuie sur un bouton :

C'était le vrai monde à l'envers et c'était la plus belle chose que j'aie vue dans ma putain de vie. A un moment, j'ai même vu Madame Rosa jeune et fraîche ... . J'en avais des larmes aux yeux. ... Et c'est là que j'ai eu un vrai événement. Je ne peux pas dire que je suis remonté en arrière et que j'ai vu ma mère, mais je me suis vu assis par terre et je voyais devant moi des jambes ..., je savais que c'était ma mère mais c'était trop tard, les souvenirs ne peuvent pas lever les yeux. J'ai même réussi à revenir encore plus loin en arrière. Je sens autour de moi deux bras chauds qui me bercent ... (121-122)

Tout comme Madame Rosa, Momo est habité par un sentiment de nostalgie perpétuelle. Cette nostalgie est liée à la perte de sa mère et à l'imminence de la mort de Madame Rosa. La possibilité de faire reculer le temps le fascine et la visualisation de ce phénomène provoque un retour en arrière inventé ou imaginaire, provenant de sa pure volonté. A travers ce recul, il opère un effacement de la souffrance et du vieillissement de Madame Rosa et parvient à se rajeunir lui-même afin de revoir sa mère. Il faut noter que le jeune Momo croit avoir 10 ans, alors qu'il a, en fait, 14 ans. Madame Rosa l'a rajeuni pour qu'il ne la quitte pas : " J'en ai jamais vraiment aimé un autre. Alors, je comptais les années et j'avais peur » (228). Dans les deux cas, le rajeunissement découle de la peur et représente une manière de rendre leur amour atemporel. Après la mort de Madame Rosa, Momo affirme : « j'ai aimé Madame Rosa et je vais continuer à la voir » (273). Dans ce texte, Gary plie et fait reculer le temps. A travers cette problématisation du temps, Gary façonne non seulement une sorte d'amour atemporel mais aussi un lignage entre ces personnages polarisés, un lignage ontologique. Le rôle du texte comme traité sur le 
Convergences francophones 6.3 (2021): 29-40

http://mrujs.mtroyal.ca/index.php/cf/index

temps et sa relation avec la nostalgie, la mort et l'amour sont constamment mis en évidence.

Du point de vue esthétique, Monsieur Ibrahim et les fleurs du Coran est un texte moins opaque. Le sens de l'humour de Schmitt évoque celui de Gary en ce qui concerne la naïveté du narrateur et s'en distingue par son côté jovial. Les premières phrases du roman lient le corps du narrateur précoce au plaisir, à l'érotisme et à l'esprit rebelle de la jeunesse : « À onze ans, j'ai cassé mon cochon et je suis allé voir les putes » (Schmitt 9). Les prostitués de Schmitt représentent une allusion subtile à Madame Rosa, ancienne prostituée, et à la communauté marginale construite par Gary. D'ailleurs, les orphelins de Madame Rosa sont presque tous des fils de prostituées et une voisine, amie chère de la famille recomposée de Madame Rosa, Madame Lola est une prostituée transgenre. Les prostituées de Schmitt, pourtant, ne se « défendent » pas, ne se battent pas, ne sont pas des personnages complexes ayant une histoire à part en tant qu'immigrée, mère ou survivante. Dans Monsieur Ibrahim et les fleurs du Coran, le corps féminin «baptise » celui du narrateur, se voit comparé à une «jolie petite chatte ... qui semble ne vivre, ne respirer, ne cligner des yeux que pour provoquer l'admiration » (17) et à une "poitrine opulente qui sa[it] si bien gémir» (22). Le personnage féminin chez Schmitt est plutôt un objet de plaisir qu'une présence pluridimensionnelle.

Toutefois, lorsqu'il s'agit du corps non-sexualisé, Schmitt imprègne le récit d'une affirmation presque constante du lien entre le corps et la joie. Les catachrèses de l'écrivain soulignent la joie intérieure de Monsieur Ibrahim dont Momo est le témoin : «J'ai jamais vu des yeux rigoler comme ça, ils rigolent à gorge déployée, ses yeux, ils font un boucan d'enfer » (18). Le pêle-mêle métaphorique redouble la naïveté du narrateur tout en esthétisant un corps harmonieux. Le sourire de Monsieur Ibrahim renvoie à celui de Monsieur Hamil, un guide spirituel de Momo dans La Vie devant soi. Le travail intertextuel de Schmitt est ici évident. La question de Momo dans La Vie devant soi («Monsieur Hamil, pourquoi vous avez toujours le sourire? »11) se voit inversée et renvoyée au jeune enfant dans Monsieur Ibrahim et les fleurs du Coran ( Pourquoi est-ce que tu ne souris jamais Momo?» 26). Dans les deux textes, le dialogue est précédé par un passage où l'enfant, Momo, explique qu'il se trouve en manque d'amour. Monsieur Hamil et Monsieur Ibrahim lient le bonheur à la quiétude spirituelle et au bien-être corporel. Dans Monsieur Ibrahim et les fleurs du Coran, Momo est convaincu que le sourire est réservé aux riches. Monsieur Ibrahim, un vieil homme qui « pass[e] pour un sage » (13), sourit tout le temps et veut que Momo soit plus heureux. "Essaie de sourire, tu verras » (27) est le conseil qu'il lui donne avant que Momo ne s'accapare de l'attention de tous, sauf de son père, avec sa nouvelle devise : «Vlan : sourire! » (27-30). Le message de Schmitt est assez constant et précis : la paix intérieure et la spiritualité créent le bonheur.

Dans La Vie devant soi, quand Momo pose la question susmentionnée, Monsieur Hamil répond: «Je remercie ainsi Dieu chaque jour pour ma bonne mémoire, mon petit Momo » (11). Ensuite, il lui raconte une histoire d'amour de jeunesse. Leurs discussions continuent à travers le récit et l'histoire d'amour 
Convergences francophones 6.3 (2021): 29-40

http://mrujs.mtroyal.ca/index.php/cf/index

constitue un leitmotiv qui exemplifie sa bonne mémoire. A la fin du récit, Monsieur Hamil devient amnésique, oubliant même son grand amour d'enfance. Or, la trajectoire de Monsieur Hamil renvoie à celle de Madame Rosa et se lie au traité sur le temps. Le message de Gary est mouvant, louvoyant entre un optimisme de Pangloss et une vision de la réalité plus voltairienne ou hugolienne. C'est un coup de génie de la part de Gary de placer entre les mains de Monsieur Hamil un exemplaire des Misérables. L'amnésie de Monsieur Hamil donne à la référence une touche sournoise lorsqu'il confond Dieu et Hugo, Momo et Victor (Hugo), Les Misérables et Le Koran. Il a toujours « son livre de Victor Hugo sous la main mais il était confusé et il croyait que c'était le Koran, car il avait les deux » (106). Il cite Les Misérables à la place du Koran. Selon le narrateur, « Monsieur Hamil devenait de plus en plus con » (106). Paradoxalement, c'est grâce à la sénilité de Monsieur Hamil que Momo semble voir Hugo et Dieu sur le même plan et se fixe l'écriture d'une grande œuvre comme but.

Les critiques de Gary soutiennent que cette inscription du nom de Hugo constitue une affirmation de la lignée entre La Vie devant soi et Les Misérables. Morin écrit : « ... le récit de La vie devant soi convoquerait la figure de l'œuvre de Victor Hugo et constituerait lui-même sa révocation. Ce roman présente de nouveaux misérables en gardant en arrière-plan les anciens » (70). Alors que l'idée d'un récit de nouveaux misérables est exprimée par Momo (218), il est apparent que cette œuvre est en fait celle qu'on lit, La Vie devant soi. Or, le rôle de Monsieur Hamil va au-delà d'un guide spirituel : il représente un testament de la part de Gary qui fait le témoignage d'une existence misérable parsemée de moments de bonheur et l'élan de la littérature. Par contraste, Monsieur Ibrahim dissimule la complexité de la pauvreté, du vieillissement et de la solitude. Monsieur Ibrahim est connu pour son sourire, il semble " échapper à l'agitation ordinaire des mortels » (13) et il meurt subitement dans un accident de voiture en bonne santé. Schmitt lui épargne le vieillissement ignoble et la démence amnésique vécus par ces doubles littéraires. Cette inversion optimiste peut être une déception pour le lecteur qui fouille les intertextes. Selon Yvonne Hsieh : «L'humanisme dont l'œuvre de Schmitt est fortement imprégnée, la concision de son style, ainsi que la présence de «formules » faciles à retenir ... sont autant de traits qui distinguent Monsieur Ibrahim et les fleurs du Coran de La Vie devant soi, et qui le rapprochent davantage d'un texte classique comme Le Petit Prince de Saint-Exupéry » (100). Bien que ce renversement soit favorable pour un lecteur qui se réjouit du chimérique, le manque de personnages 'misérables' chez Schmitt constitue une rupture de la lignée littéraire et des valeurs humanistes partagés par Hugo et Gary. Chez ces deux derniers, l'orphelin autrefois rejeté (Cosette et Momo) rend heureux son compagnon âgé (Jean Valjean et Madame Rosa) au cœur de leur souffrance extrême. Le salut qui provient de l'être laissé pour compte initie le lecteur au monde des dépourvus en lui montrant que leur soif d'amour et leur habileté à aimer ne se sont pas ternis sous le poids du malheur.

Même si les dégâts du vieillissement et de la marginalisation sont absents du texte de Schmitt, le côté psychologique de La Vie devant soi y réapparaît spectralement. Il s'agit d'une reconnaissance des conséquences à long terme de la 
Convergences francophones 6.3 (2021): 29-40

http://mrujs.mtroyal.ca/index.php/cf/index

Deuxième Guerre mondiale. Dans Monsieur Ibrahim et les fleurs du Coran, le père biologique de Momo se suicide en se jetant sous un train. C'est Monsieur Ibrahim qui lit la valeur métonymique du train et prie le jeune narrateur de le pardonner :

- Ton père, il n'avait pas d'exemple devant lui. Il a perdu ses parents très jeune parce qu'ils avaient été ramassés par les nazis et qu'ils étaient morts dans les camps. Ton père ne se remettait pas d'avoir échappé à tout ça. Peutêtre il se culpabilisait d'être en vie. Ce n'est pas pour rien qu'il a fini sous un train.

-Ah bon, pourquoi ?

- Ses parents, ils avaient été emportés par un train pour aller mourir. Lui, il cherchait peut-être son train depuis toujours... S'il n'avait pas la force de vivre, ce n'était pas à cause de toi, Momo, mais à cause de tout ce qui a été ou n'a pas été avant toi.

Puis monsieur Ibrahim m'a fourré des billets dans la poche.

-Tiens, va rue de Paradis (56) (C'est nous qui soulignons.)

Le père de Momo dans Monsieur Ibrahim et les fleurs du Coran meurt hors champ. Le lecteur n'a pas accès à ce moment crucial qui est relayé par des intermédiaires, les policiers : "Votre père est mort» (53). Ce traitement de la mort du père n'éveille point l'empathie du lecteur car Schmitt minimise cet événement jusqu'à l'abstraction. Pourtant, ce n'est pas le traitement de la mort du père qui est problématique, mais le contournement du deuil. Alors que Monsieur Ibrahim cerne de manière juste et empathique le deuil infini du père et la raison de son suicide, la réaction du fils est abrupte et frôle même l'indifférence. Après cet échange, Momo se rend chez les prostituées et refait sa vie à l'appartement en peignant les murs. Puisqu'il avait déjà vendu la bibliothèque de son père avant sa mort, il vit dorénavant dans un espace sans histoire familiale. Il s'agit d'une véritable spoliation identitaire. Or, la mort et le deuil dans ce texte ne portent qu'une valeur conceptuelle que nous interprétons logiquement par le biais de nos acquis linguistiques ou culturels. La mort du père devrait nous rendre triste, mais elle ne suscite pas plus de tristesse chez le lecteur que chez le fils. En fin de compte, la mort du père biologique permet à Momo d'échapper à une mémoire familiale traumatisante et de forger son moi selon son gré. Malheureusement, ce geste est à double tranchant car il connote aussi une rupture définitive avec la religion juive et le passé de la Shoah. Ainsi, ce geste efface en quelque sorte la mémoire des victimes dans sa famille biologique. La hantise mémorielle, qui donne au texte de Gary une triste plénitude affective et historique, est remplacée ici par la vacuité d'une réponse nonchalante («Ah bon, pourquoi ? »). Monsieur Ibrahim entrevoit le sens du geste du père, l'enfant se penche plutôt vers l'avenir et vers les plaisirs de la vie. Cette différence libère l'enfant et nie la transmission. C'est une réalité d'une époque littéraire qui veut traiter de plus en plus rapidement de la spectralité et de la hanthologie (Derrida) qui ont marqué la modernité et la postmodernité.

La mort du père de Momo dans Monsieur Ibrahim et les fleurs du Coran constitue une sorte de carrefours où plusieurs filières diégétiques de La Vie devant soi se réunissent. Tout d'abord, comme nous l'avons noté, Madame Rosa est 
Convergences francophones 6.3 (2021): 29-40

http://mrujs.mtroyal.ca/index.php/cf/index

traumatisée par sa déportation à Auschwitz durant la Deuxième Guerre mondiale et elle aussi 'cherche son train.' Schmitt semble évoquer une scène en particulier de La Vie devant soi dans laquelle Momo surprend Madame Rosa « tout habillée au milieu de la piaule à côté d'une petite valise ... et elle regard[e] à travers les murs comme si déjà elle [va] prendre le train pour toujours " (164) (C'est nous qui soulignons.) La démence de Madame Rosa déclenche le retour du refoulé et elle explique à Momo qu'elle sera transportée en Allemagne où « ils vont s'occuper de tout » (165). Les mots réverbèrent à travers le temps et l'espace : dans les deux textes, le côté intempestif du traumatisme est connoté par le mot " toujours » et le symbolique du train qui transporte les juifs vers la mort. Par ailleurs, dans les deux textes, c'est le personnage musulman qui comprend le lien entre le corps et la mémoire juive de la Shoah. Il est clair que Schmitt et Gary prônent l'empathie à l'égard de la souffrance psychologique qui va au-delà des différences culturelles, raciales et religieuses.

La mort du père dans Monsieur Ibrahim et les fleurs du Coran renvoie également à celle du père dans La Vie devant soi. Le père de Momo dans La Vie devant soi, revient voir son fils après onze ans dans un asile, punition pour le meurtre de la mère de Momo, sa 'bien-aimée' qui travaillait pour lui en tant que prostituée. L'ancien proxénète qui est maintenant mourant, anxieux et geignard « imagine des choses à cause de [s]on état psychiatrique » (192). Sachant que le père refusera un fils juif, Madame Rosa lui offre Moïse, un autre orphelin dont elle prend soin, prétendant qu'il est son fils. Le père, ahuri, accuse Madame Rosa de le persécuter. Il dit : «C'est fini le monopole juif, Madame. Il y a d'autres gens que les Juifs qui ont le droit d'être persécutés aussi. ... Je ne veux pas de fils juif sous aucun prétexte, j'ai assez d'ennuis comme ça » (196). On note que l'hostilité antisémite et l'exigence de l'homogénéité familiale sont liées à la folie d'un père meurtrier. En sortant de chez Madame Rosa, ce personnage meurt d'une crise cardiaque. On met le cadavre au bas des escaliers. Le père de Momo meurt sous le regard de son fils dans La Vie devant soi :

J'ai pincé Monsieur Yoûssef Kadir ici et là, mais il n'avait plus de problème. ... [J]e me suis assis à côté de Monsieur Yoûssef Kadir mort et je suis resté là un moment, même si on ne pouvait plus rien l'un pour l'autre. ... J'ai cherché dans ses poches pour voir s'il n'avait pas un souvenir mais il y avait seulement un paquet de cigarettes, des gauloises bleues. Il y en avait encore une à l'intérieur et je l'ai fumée assis à côté de lui, parce qu'il avait fumé toutes les autres et ça me faisait quelque chose de fumer celle qui en restait. J'ai même chialé un peu. Ça me faisait plaisir, comme s'il y avait quelqu'un à moi que j'ai perdu (201)

Dans ce passage, le jeune narrateur répète le nom du père à plusieurs reprises comme s'il le consacrait à la mémoire. Il témoigne de la mort et prend le temps de faire le deuil du père étranger. L'humour de Gary s'insinue de nouveau dans cette scène macabre lorsque l'enfant fume la cigarette volée évoquant à la fois une sorte d'héritage minable et une crémation. Les larmes du narrateur sont peu nombreuses mais couplées avec les cendres : on comprend que Momo saisit l'importance de ce 
Convergences francophones 6.3 (2021): 29-40

http://mrujs.mtroyal.ca/index.php/cf/index

moment crucial. Il est fils d'un père fou et fils/orphelin de Madame Rosa. Il est à la fois libre et héritier.

Dans Monsieur Ibrahim et les fleurs du Coran, les policiers qui annoncent le suicide du père au fils lui demandent de reconnaître le corps du défunt. Les cris de l'enfant marquent son refus. Monsieur Ibrahim convainc les policiers de lui laisser cette tâche lugubre à la place. Dans les deux textes, c'est la mort du père qui libère finalement le jeune Momo de sa langueur identitaire. Chez les deux écrivains, la mort du père représente également l'instant précis où le parent choisi par Momo affirme la nouvelle filiation, celle-ci non biologique, mais voulue. Le retour du père dans La Vie devant soi est évoqué chez Schmitt dans la scène où le suicide est annoncé par les policiers et dans une scène antérieure où la mère de Momo revient à la maison familiale qu'elle a quittée treize ans auparavant pour chercher son fils. Tout comme le père dans La Vie devant soi, les efforts de la mère sont déjoués par un jeu de noms qui constitue un refus du lignage biologique.

— Je cherche Moïse, a dit ma mère.

C'était curieux comme elle avait du mal à prononcer ce nom, comme s'il ne passait pas dans sa gorge.

Je me paie le luxe de me foutre de sa gueule.

-Vous êtes qui?

— Je suis sa mère. ... Et toi qui es-tu?

-Moi?

J'ai envie de me marrer. On n'a pas idée de se mettre dans des états pareils, surtout treize ans après.

-Moi, on m'appelle Momo.

Son visage, il se fissure.

J'ajoute en rigolant :

-C'est un diminutif pour Mohammed (58)

Chez Gary, Mohammed devient Moïse, chez Schmitt, Moïse devient Mohammed. Cette inversion cristallise le lignage littéraire entre les deux écrivains. Mohammed et Moïse, tous les deux Momo, sont frères, doubles et reflet miroir l'un de l'autre. La remise en question de l'identité nommable et statique affecte toutes les sphères de l'identité : la famille, la race, la nationalité, la religion et le langage, pour n'en citer que quelques-unes. Autrement dit, le refus du parent biologique reflète l'importance ultime du partage des valeurs humanistes, de l'empathie et de l'amour, par les membres d'une famille. Ces valeurs sont à la base du jeu intertextuel entrepris par Schmitt. Son travail de renvois et de transpositions vivifie les personnages de La Vie devant soi leur attribuant le rôle d'ancêtres choisis pour les personnages de Monsieur Ibrahim et les fleurs du Coran.

La relation entre La Vie devant soi et Monsieur Ibrahim et les fleurs du Coran fait écho à celle représentée sur la page entre le parent adoptif et le jeune garçon. Le texte originaire est plus profondément marqué par le passé et la mémoire que son héritier. La Vie devant soi marie la rupture libératrice à une pulsion nostalgique ce qui replie le temps et fait ressortir les thèmes de la nouveauté et de la mémoire. Dans Monsieur Ibrahim et les fleurs du Coran, le malheur et l'angoisse sont adoucis 
et vécus moins aigrement. Il ne s'agit pas d'un appauvrissement thématique, mais d'un changement de perspective qui place les plaisirs de la vie au centre du récit. Ce que Schmitt met en valeur à travers sa réécriture est la redéfinition de l'héritage et l'importance de la figure de l'orphelin. Dans les deux romans, l'enfant-narrateur a vécu une perte qui est survenue avant l'âge de la décision et de la responsabilité et doit alors franchir le seuil qui le sépare de l'Autre afin d'assurer sa survie. L'orphelin est le symbole de l'arrachement et de la pluralité identitaire ; son état est celui d'une ouverture constante. Il est donc d'une importance primordiale que l'orphelin choisisse de s'accrocher à un autre qui représente non la conformité et la similitude, mais l'amitié et l'amour. 


\section{Bibliographie}

Gary, Romain. La Vie devant soi. Mercure de France, 1975.

Hsieh, Yvonne. Eric-Emmanuel Schmitt, ou, La philosophie de l'ouverture. Summa Publications, 2006.

Illman, Ruth. Art and Belief : Artists Engaged in Interreligious Dialogue. Equinox, 2012.

Morin, Christian. L'Humour avec soi : analyse sémiotique du discours humoristique et de la supercherie chez Gary-Ajar. Nota bene, 2006.

Schmitt, Eric-Emmanuel. Monsieur Ibrahim et les fleurs du Coran. Albin Michel, 2001.

Viart, Dominique, et al. Le Roman français aujourd'hui. Prétexte, 2004. 